\title{
Controle de uma Formação VTNT-VANT Baseado em Espaço Nulo
}

\author{
Mauro Sérgio Mafra Moreira*, Sara Jorge e Silva* \\ Alexandre Santos Brandão** Mário Sarcinelli Filho* \\ * Programa de Pós-Graduação em Engenharia Elétrica, Universidade \\ Federal do Espirito Santo, Vitória/ES, Brasil (e-mails: \\ mauromafra@gmail.com,sarajorge.engenharia@gmail.com, \\ mario.sarcinelli@ufes.br). \\ ** Departamento de Engenharia Elétrica, Universidade Federal de \\ Viçosa,Viçosa/MG, (e-mail: alexandre.brandao@ufv.br).
}

\begin{abstract}
This paper proposes a controller to guide a formation of a differential drive wheeled robot and an aerial one to a desired state. The design adopts the paradigm of virtual structure, which is the straight line linking the two robots. The technique of null space is also adopted, splitting the whole control task in two sub-tasks, to keep the formation shape and to move the formation. The formation is described by a set of variables, the formation variables, derived from the robot positions. The formation variables are grouped into two sets, one associated to the shape of the virtual structure and the other associated to its position. The null-space technique allows giving higher priority to the sub-task of keeping the formation shape, leaving the sub-task of moving the virtual structure to a new position with a lower priority. Although being possible to give higher priority to the movement of the formation to a new position, experimental results here presented show that to give higher priority to keep the shape of the formation is the best solution, since we are interested in applications demanding to know quite well the position of the aerial robot with respect to the ground one along time, thus allowing the aerial vehicle to land over the terrestrial one.

Resumo: Este trabalho propõe um controlador para guiar dois robôs, um de tração diferencial e um aéreo, em uma tarefa de posicionamento. O projeto adota o paradigma de estrutura virtual, a qual corresponde à reta que interliga os dois robôs, e adotando a técnica de espaço nulo. Usando a técnica de espaço nulo a tarefa de controle é dividida em duas sub tarefas, manter a forma da formação e mover a formação. Neste contexto, a formação é representada por um conjunto de variáveis de formação, derivadas das posições dos robôs. As variáveis de formação são agrupadas em dois conjuntos, um associado à forma e outro à posição da estrutura virtual. Essa técnica permite estabelecer maior prioridade para uma das sub tarefas, como, por exemplo, manter a forma da formação, deixando a sub tarefa de mover a estrutura virtual com menor prioridade. Embora também se possa atribuir maior prioridade ao movimento da formação em detrimento de manter sua forma, resultados experimentais aqui apresentados mostram que atribuir maior prioridade à manutenção da forma da formação é mais vantajoso, já que visamos aplicações que exijam conhecer bem a posição do robô aéreo em relação ao terrestre ao longo do tempo, permitindo ao veículo aéreo pousar no terrestre.
\end{abstract}

Keywords: Formation control; Heterogeneous formation; Null space-based control; Shape priority; Cooperative control

Palavras-chaves: Controle de formação; Formação heterogênea; Controle baseado em espaço nulo; Prioridade de forma; Controle cooperativo

\section{INTRODUÇÃO}

Veículos aéreos não tripulados (VANTs) ganharam grande destaque em várias aplicações de robótica, muito provavelmente por causa de suas características particulares, tais como a capacidade de decolar e aterrissar verticalmente, além de possuir a capacidade de pairar sobre um ponto fixo, o que é muito importante para obter imagens durante inspeções, por exemplo.
Por outro lado, também é bem conhecido que em várias aplicações é mais eficiente ter um grupo de agentes cooperando para realizar uma tarefa, ao invés de apenas um agente. Como exemplo dessas aplicações, tem-se o transporte de carga (Pizetta et al., 2019), a manipulação de objetos (Nguyen and Garone, 2016), a busca por sobreviventes em áreas de desastres (Waharte and Trigoni, 2010) e a inspeção de plantações (Zhang and Kovacs, 2012).

Nesse contexto, surge a necessidade de controlar um grupo de agentes de forma coordenada, o que é chamado de 
controle de formação. O objetivo é controlar um pelotão de múltiplos agentes para que os mesmos se movam de maneira coordenada, resultando em um movimento de toda a formação conforme desejado.

Em relação ao controle de formação, um dos paradigmas básicos é aquele da estrutura virtual. Tal paradigma considera toda a formação como um único agente virtual (portanto sem massa, e daí sem dinâmica a ele associada). Logo, para fazer tal agente, ou seja, a formação, se mover conforme desejado os movimentos dos robôs individuais devem ser definidos (Kitts and Mas, 2009; Brandão et al., 2015). Portanto, existe uma relação entre o movimento desejado da formação e a maneira como os robôs que a compõem devem se mover, para manter a forma geométrica do pelotão.

Neste trabalho se considera uma formação de dois agentes, visando aplicações em que um veículo terrestre não tripulado (VTNT) atua em cooperação com um VANT, como num contexto de entrega de cargas, com o VANT pousando sobre uma plataforma móvel após entregar uma carga, ou até mesmo entregando a carga à plataforma móvel. Em particular, a tarefa aqui considerada corresponde ao pouso do VANT sobre o VTNT. Neste caso, é importante priorizar o controle da posição relativa dos agentes para um melhor desempenho na realização da tarefa. Por este motivo, o paradigma de estrutura virtual é a abordagem mais adequada nesta situação (Rabelo et al., 2018).

Para a aplicação aqui considerada, então, é necessário estar atento às posições relativas dos agentes: para pousar no VTNT o VANT deve estar, necessariamente, sobrevoando o mesmo. Como é descrito adiante, a formação utilizada é caracterizada por um conjunto de variáveis, parte delas associada à sua forma, e parte delas associada à sua posição. Neste contexto, as variáveis associadas à forma da formação serão tratadas separadamente das variáveis associadas à posição da formação. Portanto, a tarefa de controlar o conjunto de agentes é desmembrada em duas sub-tarefas, sendo a primeira controlar a forma da formação e a segunda controlar sua posição. Neste trabalho é atribuída maior prioridade ao controle da forma da formação, visando maior precisão quanto à posição relativa dos dois agentes, sendo tal priorização implementada utilizando-se a técnica de espaço nulo (Antonelli et al., 2010; Rosales et al., 2016).

Finalmente, para validar experimentalmente o sistema de controle proposto, uma formação composta por um robô de tração diferencial Pioneer 3-DX e um quadrimotor Parrot AR.Drone 2.0 é considerada (ver Figura 1). As posições dos dois robôs são fornecidas por um sistema OptiTrack de captura de movimento, composto por quatro câmeras, o qual é executado em um computador externo à formação. Tal computador também é usado para executar o sistema de controle, caracterizando assim uma arquitetura de controle centralizada. Em outras palavras, tal computador executa o sistema OptiTrack, a partir do qual obtém as posições do VTNT e do VANT, executa o algoritmo de controle, obtendo os comandos a serem enviados aos dois veículos, e envia tais comandos aos mesmos através de um link Wi-Fi, concluindo assim um ciclo de controle.

Para lidar com esses tópicos, este artigo é dividido em algumas seções. A partir deste ponto a Seção 2 detalha a descrição da formação e suas variáveis, bem como a relação entre as variáveis de formação e as posições dos agentes individuais. Na sequência, na Seção 3 é descrita a técnica de espaço nulo, enquanto a Seção 4 descreve como tal técnica é integrada ao sistema de controle proposto. Prosseguindo, na Seção 5 são apresentados resultados experimentais que validam o sistema de controle proposto. Por fim, a Seção 6 destaca as principais conclusões do trabalho.

\section{CARACTERIZAÇÃO DA FORMAÇÃO}

A Figura 2 mostra um esboço descritivo da formação utilizada, incluindo os sistemas de coordenadas global, do VTNT e do VANT, e a caracterização das variáveis envolvidas. A formação pode ser entendida como a linha reta ligando os dois veículos, e é representada na figura como a linha contínua cujo comprimento é $\rho_{f}$, que começa na posição $\left[\begin{array}{lll}x_{f} & y_{f} & z_{f}\end{array}\right]^{T}=\left[\begin{array}{lll}x_{1} & y_{1} & z_{1}\end{array}\right]^{T}$ (a posição do VTNT), forma o ângulo $\beta_{f}$ com o plano $X Z$, e cuja projeção sobre esse plano forma o ângulo $\alpha_{f}$ com eixo $X$. Observe-se que a posição da formação é caracterizada pela posição do VTNT, porque a ideia é que o VANT possa pousar no VTNT, precisando, para isso, posicionar-se exatamente acima dele. Note-se, também, que a coordenada $z_{1}$ do VTNT está sendo considerada, mas é mantida em zero o tempo todo neste trabalho (a inclusão de tal coordenada na formulação do problema visa permitir generalizar a formação para envolver dois VANTs). Tal configuração também poderia ser adotada para uma formação de dois VTNTs, fazendo-se $z_{1}=0 \mathrm{~m}, z_{2}=0 \mathrm{~m}$ e $\beta_{f}=0^{\circ}$.

A partir de tal figura se estabelecem as relações entre as variáveis de formação $\mathbf{q}=\left[\begin{array}{llllll}x_{f} & y_{f} & z_{f} & \rho_{f} & \beta_{f} & \alpha_{f}\end{array}\right]^{T}$ e as posições dos dois robôs, dadas pelo vetor $\mathbf{x}=\left[\mathbf{h}_{1}^{T} \mathbf{h}_{2}^{T}\right]^{T}=$

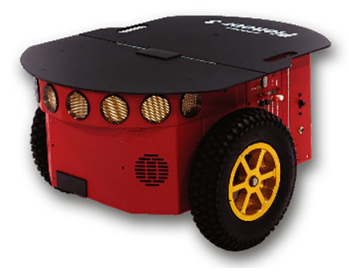

(a) VTNT (Pioneer3-DX)

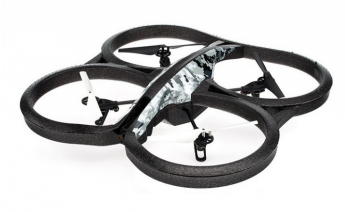

(b) VANT (AR.Drone 2.0)
Figura 1. Os dois robôs utilizados na formação.

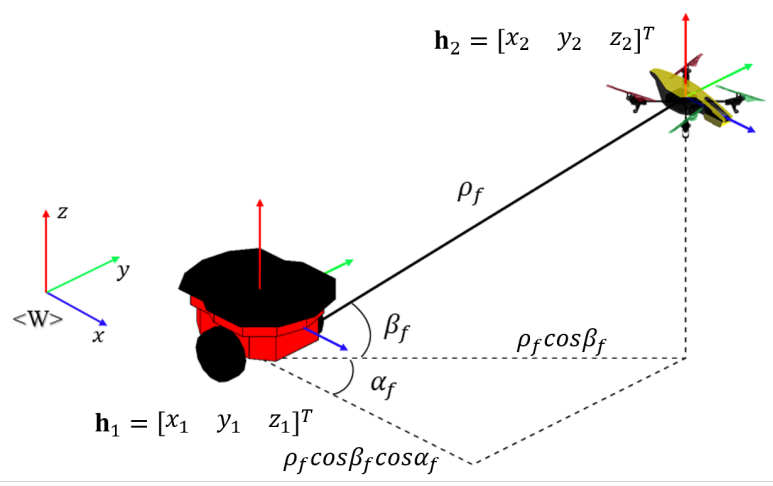

Figura 2. A caracterização da formação linear composta por um Pioneer 3-DX e um AR.Drone 2.0. 
$\left[\begin{array}{llllll}x_{1} & y_{1} & z_{1} & x_{2} & y_{2} & z_{2}\end{array}\right]^{T}$, onde $\mathbf{h}_{1}$ e $\mathbf{h}_{2}$ representam as posições do VTNT e do VANT, respectivamente. Se descrevem, então, uma transformação das variáveis dos robôs para as variáveis de formação $(\mathbf{x} \rightarrow \mathbf{q})$ e outra das variáveis de formação para as variáveis dos robôs $(\mathbf{q} \rightarrow \mathbf{x})$. São elas

para a qual

$$
\mathbf{q}=f(\mathbf{x})
$$

$$
\begin{gathered}
x_{f}=x_{1}, y_{f}=y_{1}, z_{f}=z_{1}, \rho_{f}=\sqrt{d_{x}^{2}+d_{y}^{2}+d_{z}^{2}}, \\
\alpha_{f}=\tan ^{-1}\left(\frac{d_{y}}{d_{x}}\right), \text { e } \beta_{f}=\tan ^{-1}\left(\frac{d_{z}}{\sqrt{d_{x}^{2}+d_{y}^{2}}}\right), \\
\text { sendo } d_{x}=x_{2}-x_{1}, d_{y}=y_{2}-y_{1}, \text { e } d_{z}=z_{2}-z_{1}, \mathrm{e} \\
\mathbf{x}=f^{-1}(\mathbf{q}),
\end{gathered}
$$

para a qual

$$
\begin{gathered}
x_{1}=x_{f}, y_{1}=y_{f}, z_{1}=z_{f}, x_{2}=x_{f}+\rho_{f} \cos \left(\alpha_{f}\right) \cos \left(\beta_{f}\right), \\
y_{2}=y_{f}+\rho_{f} \sin \left(\alpha_{f}\right) \cos \left(\beta_{f}\right), \text { e } z_{2}=z_{f}+\rho_{f} \sin \left(\beta_{f}\right) .
\end{gathered}
$$

Usando tais equações pode-se descrever como cada robô deve se mover para alterar a formação conforme desejado, e como a formação muda com o movimento dos dois robôs. Logo, especificando como a formação deve se mover se define como cada robô deve se mover para realizar o movimento desejado da formação. Portanto, qualquer controlador proposto para guiar a formação deve agir sobre os robôs individuais para controlar seu movimento, garantindo que a formação como um todo se mova para a posição desejada, mantendo sua forma.

Rabelo et al. (2018) propõem um esquema de controle para uma formação idêntica àquela aqui considerada, para fazêla percorrer um grupo de waypoints: o VTNT vai para cada um desses waypoints, que descrevem a posição desejada da formação em determinados momentos, e o VANT deve acompanhá-lo, mantendo a forma da formação conforme especificado pelos valores de $\rho_{f}, \beta_{f}$ e $\alpha_{f}$. Porém, o esquema de controle ali proposto considera o controle da posição da formação (caracterizada por $x_{f}, y_{f}$ e $z_{f}$ ) e o controle da forma da formação (caracterizada por $\rho_{f}, \beta_{f}$ e $\alpha_{f}$ ) com a mesma prioridade. Neste trabalho, ao contrário, considerando que o VANT deve pousar no VTNT mesmo se este se desviar de seu caminho (para evitar um obstáculo em seu caminho, mas que não é um obstáculo para o VANT, por exemplo), o sistema de controle atribui maior prioridade para a manutenção da forma da formação. Para fazer isso, as variáveis de formação são divididas em dois conjuntos, um correspondente às variáveis de posição da formação $\left(\left[\begin{array}{lll}x_{f} & y_{f} & z_{f}\end{array}\right]^{T}\right)$ e o outro correspondente às variáveis de forma da formação $\left(\left[\begin{array}{lll}\rho_{f} & \alpha_{f} & \beta_{f}\end{array}\right]^{T}\right)$, e o controlador a ser projetado deve considerar que o controle das variáveis de forma tem maior prioridade em relação ao controle das variáveis de posição. Para realizar tal hierarquização é adotada a técnica de espaço nulo (Antonelli et al., 2010; Rosales et al., 2016).

\section{CONTROLE BASEADO EM ESPAÇO NULO}

A abordagem utilizando a metodologia de espaço nulo pode ser considerada como uma maneira analítica de implementar controle baseado em comportamento para sistemas multi-robôs. Tal abordagem permite executar tarefas diferentes, mesmo que elas sejam conflitantes. A ideia geral é dividir a tarefa a ser executada pela formação em algumas sub-tarefas complementares, que devem ser executadas obedecendo a uma certa hierarquia Antonelli et al. (2010).

Considerando a formação aqui tratada, a missão a ser cumprida é levar dois robôs de suas posições iniciais, cada um para sua posição desejada, mantendo uma formação específica ao longo da navegação, ou seja, compondo a linha reta correspondente à estrutura virtual desejada. Assim, o conjunto de valores iniciais das variáveis dos robôs $\mathbf{x}$ e o conjunto de variáveis de formação q desejadas compõem o conjunto de variáveis de entrada e devem ser especificadas. Em seguida, duas tarefas parciais podem ser identificadas, a saber: 1 - manter a forma da formação (controlando o vetor $\mathbf{q}_{f}=\left[\begin{array}{lll}\rho_{f} & \alpha_{f} & \beta_{f}\end{array}\right]^{T}$ ), e 2 - controlar a posição da formação (controlando o vetor $\mathbf{q}_{p}=\left[\begin{array}{lll}x_{f} & y_{f} & z_{f}\end{array}\right]^{T}$ ). Os controladores são projetados considerando a execução independente das tarefas, e os sinais de controle gerados pelas duas tarefas são então combinados para obter as referências de movimento para os robôs. A maneira de combinar as diferentes tarefas parciais será implementada com base em hierarquia, conforme detalhado a seguir.

Note-se que ao particionar o controle em duas partes, como acima mencionado, os dois subsistemas ficam redundantes. Isso significa que são usados seis graus de liberdade $(\mathbf{x})$ para controlar três graus de liberdade $\left(\mathbf{q}_{p}\right.$ ou $\left.\mathbf{q}_{f}\right)$.

Uma outra abordagem para controlar o movimento da formação é considerar toda a tarefa de uma só vez, ou seja, controlar todo o vetor $\mathbf{q}=\left[\begin{array}{llllll}x_{f} & y_{f} & z_{f} & \rho_{f} & \alpha_{f} & \beta_{f}\end{array}\right]^{T}$, como em (Rabelo et al., 2018) (nesse caso tem-se um sistema completamente atuado). Já o projeto do controlador aqui proposto começa considerando a matriz jacobiana correspondente a (1), dada por $\mathbf{J}(\mathbf{x})$ em

$$
\dot{\mathbf{q}}=\mathbf{J}(\mathbf{x}) \dot{\mathbf{x}}
$$

Tal matriz é uma matriz $6 \times 6$, definida como

$$
\mathbf{J}(\mathbf{x})=\frac{\partial \mathbf{q}}{\partial \mathbf{x}}=\left[\begin{array}{cccc}
\frac{\partial x_{f}}{\partial x_{1}} & \frac{\partial x_{f}}{\partial y_{1}} & \cdots & \frac{\partial x_{f}}{\partial z_{2}} \\
\frac{\partial y_{f}}{\partial x_{1}} & \frac{\partial y_{f}}{\partial y_{1}} & \cdots & \frac{\partial y_{1}}{\partial z_{2}} \\
\vdots & \vdots & \ddots & \vdots \\
\frac{\partial \alpha_{f}}{\partial x_{1}} & \frac{\partial \alpha_{f}}{\partial y_{1}} & \cdots & \frac{\partial \alpha_{f}}{\partial z_{2}}
\end{array}\right]
$$

o que, no caso presente, corresponde a

$$
\mathbf{J}(\mathbf{x})=\left[\begin{array}{cccccc}
1 & 0 & 0 & 0 & 0 & 0 \\
0 & 1 & 0 & 0 & 0 & 0 \\
0 & 0 & 1 & 0 & 0 & 0 \\
\frac{d_{x}}{r} & \frac{d_{y}}{r} & \frac{d_{z}}{r} & \frac{-d_{x}}{r} & \frac{-d_{y}}{r} & \frac{-d_{z}}{r} \\
\frac{-d_{y}}{d^{2}} & \frac{d_{x}}{d^{2}} & 0 & \frac{d_{y}}{d^{2}} & \frac{-d_{x}}{d^{2}} & 0 \\
\frac{d_{x} d_{z}}{r^{2} d} & \frac{d_{y} d_{z}}{r^{2} d} & \frac{-d}{r^{2}} & \frac{-d_{x} d_{z}}{r^{2} d} & \frac{-d_{y} d_{z}}{r^{2} d} & \frac{d}{r^{2}}
\end{array}\right]
$$


Para controlar como a formação varia no tempo, ou seja, $\dot{\mathbf{q}}$, adota-se simplesmente um controlador de cinemática inversa, já que a formação é um robô virtual, logo sem massa. Assim, considerando as variáveis de formação desejadas $\mathbf{q}_{d e s}$, sua variação no tempo $\dot{\mathbf{q}}_{d e s}$, e o erro $\tilde{\mathbf{q}}=\mathbf{q}_{d e s}-\mathbf{q}$, pode-se usar a lei de controle

$$
\mathbf{v}_{\text {ref }}=\mathbf{J}^{-1}(\mathbf{x})\left[\dot{\mathbf{q}}_{\text {des }}+\mathbf{k}_{1} \tanh \left(\mathbf{k}_{2} \tilde{\mathbf{q}}\right)\right]
$$

para gerar as velocidades desejadas para os robôs individuais, onde $\mathbf{k}_{1}$ e $\mathbf{k}_{2}$ são matrizes de ganho/saturação diagonais e positivas definidas. Ressalte-se que o sistema de controle de malha fechada, adotando tal lei de controle, é estável, conforme provado em (Rabelo et al., 2018).

Separando-se a tarefa de controle global nas duas subtarefas correspondentes ao controle da forma e ao controle da posição da formação, associados às variáveis de formação $\mathbf{q}_{p}$ e $\mathbf{q}_{f}$, se definem duas leis de controle, que são

$$
\begin{gathered}
\mathbf{v}_{\text {fref }}=\mathbf{J}_{f}^{\#}(\mathbf{x})\left[\dot{\mathbf{q}}_{\text {fdes }}+\mathbf{k}_{1 f} \tanh \left(\mathbf{k}_{2 f} \tilde{\mathbf{q}}_{f}\right)\right], \mathrm{e} \\
\mathbf{v}_{\text {pref }}=\mathbf{J}_{p}^{\#}(\mathbf{x})\left[\dot{\mathbf{q}}_{\text {pdes }}+\mathbf{k}_{1 p} \tanh \left(\mathbf{k}_{2 p} \tilde{\mathbf{q}}_{p}\right)\right],
\end{gathered}
$$

onde $\mathbf{J}_{p}$ e $\mathbf{J}_{f}$ são matrizes não quadradas obtidas particionando $\mathbf{J}(\mathbf{x}), \mathbf{J}_{p}$ correspondendo às três primeiras linhas de $\mathbf{J}(\mathbf{x})$ e $\mathbf{J}_{f}$ correspondendo às últimas três linhas de $\mathbf{J}(\mathbf{x})$, com $\mathbf{k}_{1 f}, \mathbf{k}_{1 p}, \mathbf{k}_{2 f}, \mathbf{k}_{2 p}, \mathbf{k}_{1}$ e $\mathbf{k}_{2}$ sendo matrizes de ganho/saturação diagonais e positivas definidas. Quanto a $\mathbf{J}_{p}^{\#}$ e $\mathbf{J}_{f}^{\#}$, elas são as pseudo-inversas Moore-Penrose de $\mathbf{J}_{p}$ e $\mathbf{J}_{f}$. A função tanh(.), por sua vez, é usada como uma função de saturação suave, para evitar que grandes valores de $\tilde{\mathbf{q}}$ gerem sinais de controle inviáveis para os robôs.

Depois de obter as leis de controle para as duas sub-tarefas, existem duas maneiras de compor o sinal de controle resultante: a primeira é dar a maior prioridade à tarefa de manter a forma da formação, deixando a sub tarefa de controlar a posição da formação com menor prioridade, enquanto a segunda é exatamente o inverso. Neste trabalho o objetivo é dar a maior prioridade à sub tarefa de controlar a forma da formação, pois o interesse é mover a formação de forma a garantir o pouso do VANT no VTNT. Assim é que este trabalho compara o desempenho do controlador baseado no espaço nulo de $\mathbf{J}_{f}^{\#}$ contra aqueles do controlador de Rabelo et al. (2018) e do controlador baseado em espaço nulo de $\mathbf{J}_{p}^{\#}$, correspondente a atribuir a prioridade mais alta ao controle da posição da formação.

\section{O CONTROLADOR PROPOSTO}

Atribuir menor prioridade a uma certa sub tarefa é projetá-la no espaço nulo da tarefa com prioridade imediatamente acima, para a tarefa de menor prioridade só ser executada quando não conflitar com aquela. No caso presente isso corresponde a fazer (Antonelli et al., 2010)

$$
\mathbf{v}_{\text {ref }}=\mathbf{v}_{\text {fref }}+\left(\mathbf{I}-\mathbf{J}_{f}^{\#} \mathbf{J}_{f}\right) \mathbf{v}_{\text {pref }},
$$

para atribuir a prioridade mais alta ao controle da forma da formação, ou

$$
\mathbf{v}_{\text {ref }}=\mathbf{v}_{\text {pref }}+\left(\mathbf{I}-\mathbf{J}_{p}^{\#} \mathbf{J}_{p}\right) \mathbf{v}_{\text {fref }},
$$

para atribuir a prioridade mais alta ao controle da posição da formação (no primeiro caso, obtém-se uma formação rígida, enquanto que no segundo caso se obtém uma formação flexível).

Para o primeiro caso, o sistema de controle, referente à formação de um VTNT e um VANT, é aquele mostrado na Figura 3, que é o sistema de controle que propomos para guiar o VTNT e o VANT e permitir o pouso do segundo no primeiro. Para implementar o segundo caso, basta alterar os índices $f$ para $p$ e $p$ para $f$ no diagrama. Quanto à análise de estabilidade desses dois controladores, o leitor deve consultar (Rosales et al., 2016).

\section{RESULTADOS}

Nesta seção o controlador da Figura 3, doravante denominado Controle NSB com Prioridade de Forma, é adotado para guiar a formação em uma tarefa de posicionamento. Assim, temos os valores $\dot{\mathbf{q}}_{\text {des }}=\mathbf{0}$, assim como $\dot{\mathbf{x}}_{d e s}$ e $\ddot{\mathbf{x}}_{\text {des }}$. Observe-se que o mesmo controlador também pode ser usado para executar tarefas de seguimento de trajetória. Para isso $\dot{\mathbf{q}}_{d e s}$ deve ser função do tempo (a trajetória a ser rastreada) e sua derivada no tempo $\dot{\mathbf{q}}_{d e s}$ também é considerada. Quanto a $\mathbf{x}_{\text {des }}$, tal vetor é definido usando (2), enquanto $\dot{\mathbf{x}}_{\text {des }}$ é obtido derivando $\mathbf{x}_{\text {des }}$ no tempo.

A formação aqui utilizada é composta por dois robôs reais, um Pioneer 3-DX e um quadrimotor Parrot AR.Drone 2.0. Além do controlador da Figura 3 outros dois controladores são também implementados e testados utilizando tal formação, para fins de comparação de desempenho, a saber o controlador proposto em (Rabelo et al., 2018), daqui em diante denominado Controle Convencional, que considera a lei de controle em (5), e a configuração semelhante à da Figura 3 que implementa a lei de controle em (9), daqui em diante denominada Controle NSB com Prioridade de Posição. O objetivo é comparar o desempenho de tais controladores, para verificar, por exemplo, se dar maior prioridade para manter a forma da formação provoca ou não perda de desempenho.

As posições iniciais dos dois robôs da formação são $\mathbf{h}_{1_{0}}=\left[\begin{array}{lllll}-1 & m-1 & m & 0 & m\end{array}\right]$, para o Pioneer 3-DX, e $\mathbf{h}_{2_{0}}=$ [-1 $m 1 \mathrm{~m} 1 \mathrm{~m}$ ], para o AR.Drone 2.0. Quanto à posição e forma desejadas para a formação, elas são $\mathbf{q}_{d e s}=$ $[0 \mathrm{~m} 1 \mathrm{~m} 0 \mathrm{0} 1 \mathrm{~m} \mathrm{pi} / 3 \mathrm{rad} 0 \mathrm{rad}]$. Como se trata de uma tarefa de posicionamento, $\dot{\mathbf{q}}_{\text {des }}=\mathbf{0}$ e $\dot{\mathbf{x}}_{\text {des }}=\mathbf{0}$ (ver Figura 3). Finalmente, as matrizes de ganho associadas aos controladores são $\mathbf{k}_{1 p}=\operatorname{diag}\left[\begin{array}{lll}1 & 1 & 0\end{array}\right], \mathbf{k}_{1 f}=$ $\operatorname{diag}\left[\begin{array}{lll}1 & 0.25 & 0.35\end{array}\right], \mathbf{k}_{2 p}=\operatorname{diag}\left[\begin{array}{lll}0.25 & 0.1 & 0.1\end{array}\right]$, e $\mathbf{k}_{2 f}=$ $\operatorname{diag}\left[\begin{array}{lll}0.1 & 0.25 & 0.2\end{array}\right]$. Note-se que $\mathbf{k}_{1 p}(3,3)=0$ porque estamos considerando que $z_{f}=z_{1}=0$ todo o tempo, já que tal coordenada se refere ao VTNT. Por sua vez, $\mathbf{k}_{1}=\operatorname{diag}\left[\mathbf{k}_{1 p} \mathbf{k}_{1 f}\right]$ e $\mathbf{k}_{2}=\operatorname{diag}\left[\mathbf{k}_{2 p} \mathbf{k}_{2 f}\right]$.

Os trajetos dos robôs no experimento podem ser vistos nas Figuras 4, 5 e 6, que se referem ao Controle Convencional, ao Controle NSB com Prioridade de Forma, e ao Controle NSB com Prioridade de Posição, respectivamente.

Os resultados do experimento evidenciam uma diferença significativa de desempenho entre os controladores, como se pode ver comparando os gráficos das Figuras 7, 8 e 9. Os ângulos que caracterizam a formação são menos estáveis para o Controle Convencional, em comparação com os do Controle NSB com Prioridade de Posição e do Controle NSB com Prioridade de Forma, especialmente este último. 


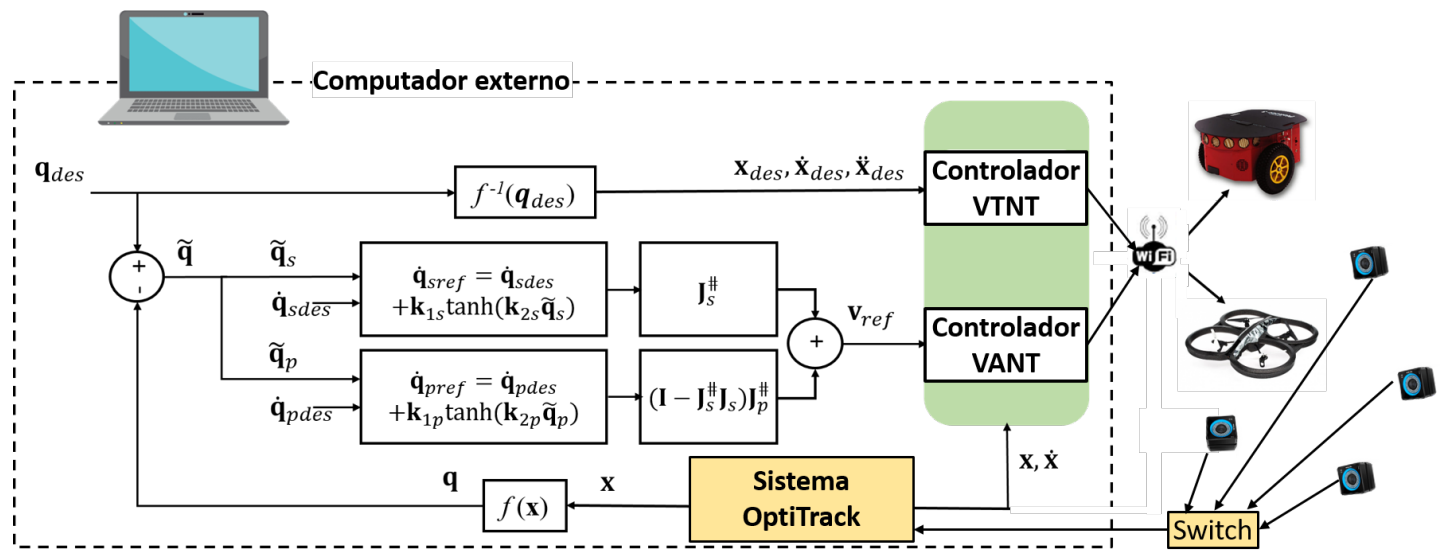

Figura 3. Diagrama de blocos para o controlador proposto, que atribui maior prioridade ao controle da forma da formação.

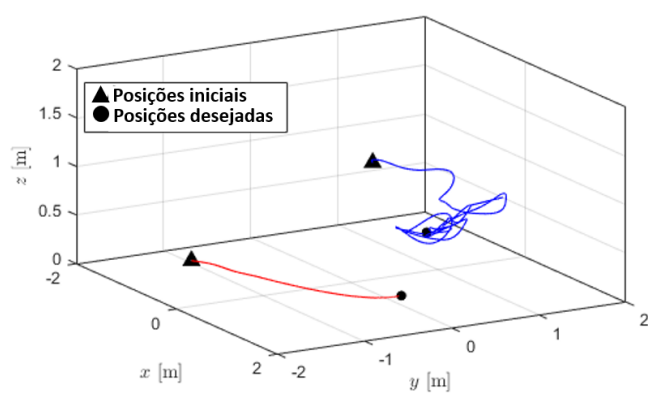

Figura 4. Percursos dos robôs no Controle Convencional.

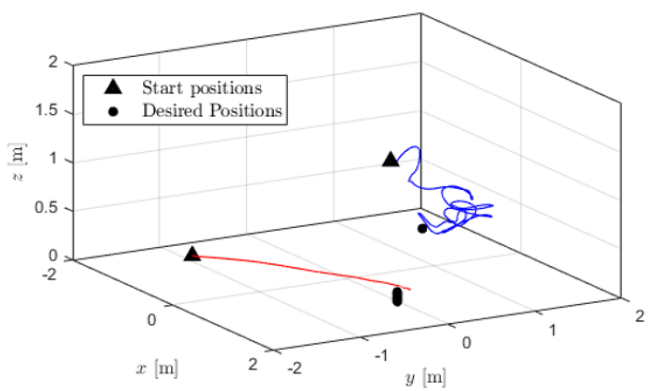

Figura 5. Percursos dos robôs no Controle NSB com Prioridade de Forma.

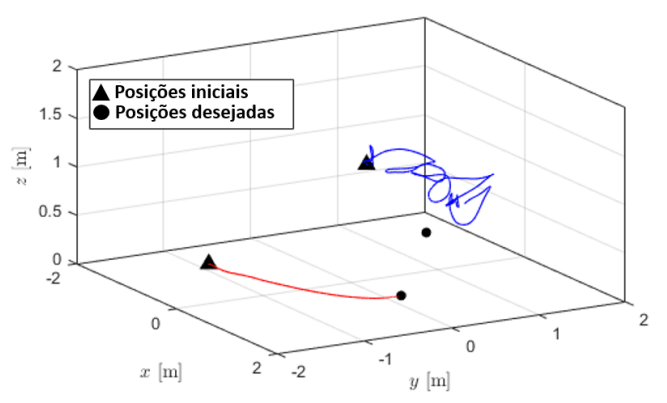

Figura 6. Percursos dos robôs no Controle NSB com Prioridade de Posição.

Observando a Figura 7, o caso do Controle Convencional apresenta alta variação de ângulos para os parâmetros adotados, embora todos os erros tendam a zero ao longo do tempo. Quando manter a forma da formação é a prioridade mais alta, ou seja, no Controle NSB com Prioridade de Forma, ambos os robôs primeiro procuram um ao outro, tentando reduzir os erros de forma. Ambos os objetivos, reduzir os erros de forma e de posição, são buscados, mas o controlador prioriza a manutenção do formato da formação.

No caso do Controle NSB com Prioridade de Posição (ver Figura 9) o controlador permite mais aceleração, o que causa maiores erros de forma. Ou seja, o controlador está tentando reduzir o erro de posição o mais rápido possível, mesmo à custa de erros de forma maiores.

Em resumo, todos os três controladores fazem os erros de forma e posição tenderem a zero ao longo do tempo. Mas se o foco principal é manter a forma da formação, o Controle NSB com Prioridade de Forma traz melhores resultados, como mostram os resultados do experimento. Como exemplo, suponha que o VTNT mude seu caminho para evitar um obstáculo quando o VANT estiver tentando pousar nele. Nesse caso, a melhor opção seria certamente usar o Controle NSB com Prioridade de Forma aqui discutido, para que se tenha maior confiança que o VANT estará em condição de pousar no VTNT.
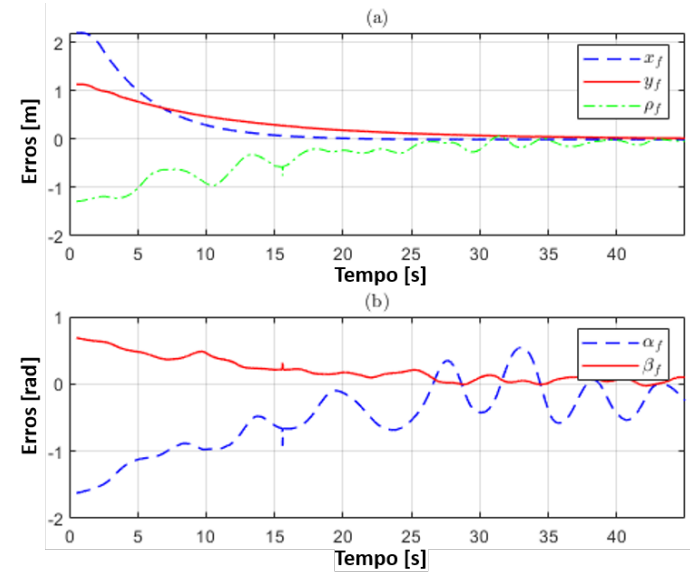

Figura 7. Erros para o Controle Convencional. 

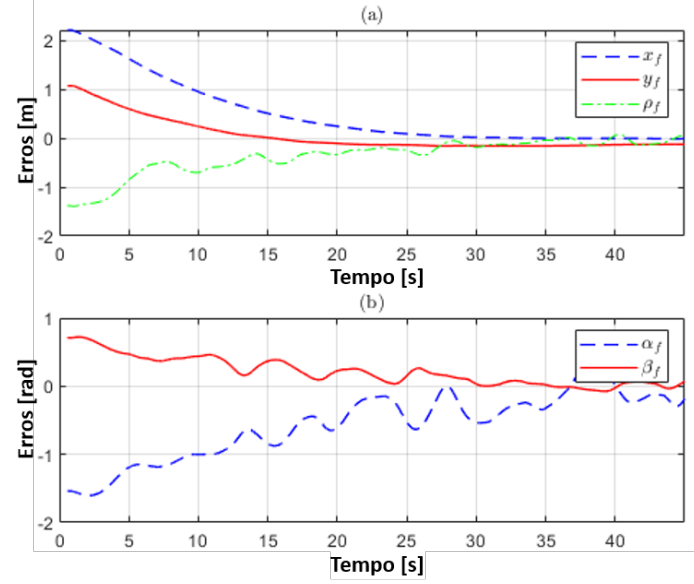

Figura 8. Erros para o Controle NSB com Prioridade de Forma.
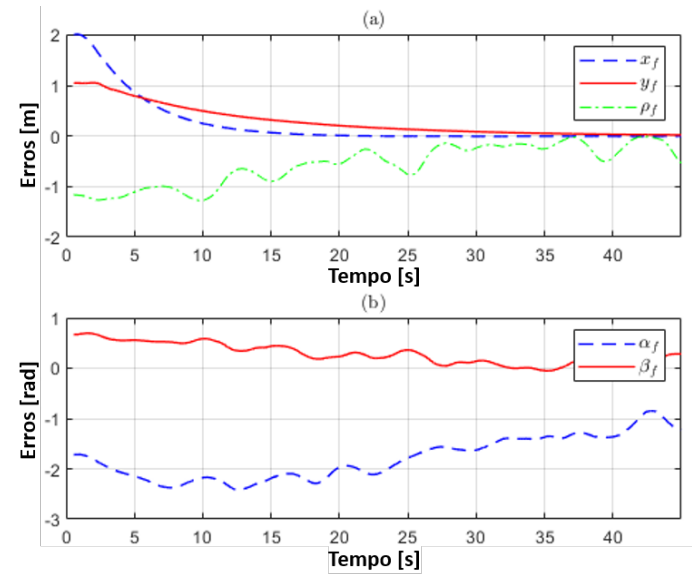

Figura 9. Erros para o Controle NSB com Prioridade de Posição.

\section{CONCLUSÃO}

Neste trabalho um sistema de controle foi proposto para conduzir a formação de um VTNT e um VANT, visando o pouso do VANT no VTNT em movimento. O VANT deve manter uma certa posição em relação ao VTNT, para então aterrissar nele. A proposta consiste em controlar a formação VTNT-VANT associando-a a uma estrutura virtual, que é a linha reta ligando os dois veículos. Para permitir que o VANT aterrisse no VTNT, a estrutura virtual deve manter a forma desejada (comprimento $\rho_{F} \mathrm{e}$ ângulos $\beta_{F}$ em relação ao eixo $Z$ e $\alpha_{F}$ da projeção de linha sobre o plano $X Y$ e o eixo $X$ ) o mais próximo possível dos valores especificados. A formação é descrita a partir da posição do VTNT (o vetor $\left[\begin{array}{lll}x_{1} & y_{1} & z_{1}\end{array}\right]^{T}$ ), cuja posição define a posição da formação (o vetor $\left[\begin{array}{lll}x_{F} & y_{F} & z_{F}\end{array}\right]^{T}$ ), cuja forma (variáveis $\rho_{F}, \beta_{F}, \alpha_{F}$ ) está relacionada às posições do VTNT e do VANT (vetor $\left[\begin{array}{lll}x_{2} & y_{2} & z_{2}\end{array}\right]^{T}$ ).

Para assegurar que os erros nas variáveis de forma associadas à formação ao longo do seu movimento sejam mantidos em valores baixos, a técnica de controle baseada no espaço nulo é adotada, atribuindo-se maior prioridade ao controle de forma da formação. A ideia é que o VANT possa pousar no VTNT mesmo que a posição deste inclua grandes erros.
Finalmente, o esquema de controle proposto é validado através de resultados experimentais, após comparações com outros dois controladores similares.

Como próximo passo do trabalho, pretende-se aplicar o esquema de controle baseado em espaço nulo com prioridade de forma em experimentos nos quais o VANT pousa sobre o VTNT, inclusive em situações onde o VTNT está executando manobras para desviar-se de algum obstáculo no seu caminho.

Num horizonte mais longo, também se expandirá o sistema de controle proposto para situações envolvendo mais agentes. A ideia é aplicar o sistema aqui proposto para cada par de agentes, decompondo-se a formação de mais que dois agentes em diversas formações de dois agentes, sejam tais formações parciais compostas por dois VTNTs, por um VTNT e um VANT, ou mesmo por dois VANTs.

\section{AGRADECIMENTOS}

Os autores agradecem ao CNPq - Conselho Nacional de Desenvolvimento Científico e Tecnológico, agência de fomento do Ministério da Ciência, Tecnologia, Inovações e Comunicações, e à FAPES - Fundação de Amparo à Pesquisa e Inovação do Espírito Santo, agência de fomento do Estado do Espírito Santo, pelo suporte financeiro que permitiu desenvolver este trabalho.

\section{REFERÊNCIAS}

Antonelli, G., Arrichiello, F., and Chiaverini, S. (2010). The nsb control: a behavior-based approach for multirobot systems. Paladyn, 1(1), 48-56.

Brandão, A.S., Rampinelli, V.T.L., Martins, F.N., Sarcinelli-Filho, M., and Carelli, R. (2015). The multilayer control scheme: A strategy to guide $n$-robots formations with obstacle avoidance. Journal of Control, Automation and Electrical Systems, 26(3), 201-214.

Kitts, C.A. and Mas, I. (2009). Cluster space specification and control of mobile multirobot systems. IEEE/ASME Transactions on Mechatronics, 14(2), 207-218.

Nguyen, T. and Garone, E. (2016). Control of a uav and a ugv cooperating to manipulate an object. In American Control Conference (ACC), 2016, 1347-1352. IEEE.

Pizetta, I.H.B., Brandão, A.S., and Sarcinelli-Filho, M. (2019). Avoiding obstacles in cooperative load transportation. ISA Transactions (online access).

Rabelo, M.F.S., Brandão, A.S., and Sarcinelli-Filho, M. (2018). Centralized control for an heterogeneous line formation using virtual structure approach. In 2018 Latin American Robotic Symposium (LARS), 135-140. João Pessoa, PB, Brazil.

Rosales, C., Leica, P., Sarcinelli-Filho, M., Scaglia, G., and Carelli, R. (2016). 3d formation control of autonomous vehicles based on null-space. Journal of Intelligent \& Robotic Systems, 84(1-4), 453-467.

Waharte, S. and Trigoni, N. (2010). Supporting search and rescue operations with uavs. In 2010 International Conference on Emerging Security Technologies, 142147.

Zhang, C. and Kovacs, J.M. (2012). The application of small unmanned aerial systems for precision agriculture: a review. Precision Agriculture, 13(6), 693-712. 